\title{
Immunogenicity and Safety of a Tetravalent Recombinant Subunit Dengue Vaccine in Adults Previously Vaccinated with a Live Attenuated Tetravalent Dengue Vaccine: Results of a Phase-I Randomized Clinical Trial
}

\begin{abstract}
Anna P. Durbin, ${ }^{1}$ Kristen K. Pierce,,${ }^{2,3}$ Beth D. Kirkpatrick, ${ }^{2,3}$ Palmtama Grier, ${ }^{1}$ Beulah P. Sabundayo, ${ }^{1}$ Helen He, ${ }^{1}$ Michele Sausser, ${ }^{4}$ Amy Falk Russell, ${ }^{4}$ Jason Martin, ${ }^{4}$ Donna Hyatt, ${ }^{4}$ Melissa Cook, ${ }^{4}$ Jeffrey R. Sachs, ${ }^{4}$ Andrew Wen-Tseng Lee, ${ }^{4}$ Liman Wang, ${ }^{4}$ Beth-Ann Coller, ${ }^{4}$ and Stephen S. Whitehead ${ }^{5 *}$

${ }^{1}$ Department of International Health, Center for Immunization Research, Johns Hopkins Bloomberg School of Public Health, Baltimore, Maryland; ${ }^{2}$ Department of Microbiology and Molecular Genetics, Vaccine Testing Center, Larner College of Medicine, University of Vermont, Burlington, Vermont; ${ }^{3}$ Department of Medicine, Vaccine Testing Center, Larner College of Medicine, University of Vermont, Burlington, Vermont; ${ }^{4}$ Merck \& Co., Inc., Kenilworth, New Jersey; ${ }^{5}$ Laboratory of Viral Diseases, National Institute of Allergy and Infectious Diseases, National Institutes of Health, Bethesda, Maryland
\end{abstract}

\begin{abstract}
New dengue vaccines are needed to prevent this globally expanding vector-borne disease. The V180 vaccine candidate consists of four recombinant, soluble, dengue virus envelope glycoproteins and has been previously evaluated in two clinical trials for safety and immunogenicity in Flavivirus-naive participants (NCT01477580 and NCT0093642). Here, we report on a randomized, placebo-controlled, double-blind study of the safety and immunogenicity of the V180 vaccine in subjects who have previously received the live attenuated tetravalent vaccine (LATV) developed by the National Institute of Allergy and Infectious Diseases (protocol \#V180-002 [CIR-301]). The study was designed to evaluate whether this recombinant subunit vaccine could boost the neutralizing antibody responses induced by dengue LATV. Twenty participants who had previously received one or two doses of dengue LATV were randomized and received a single dose of $\mathrm{V} 180$ nonadjuvanted $(N=8), \mathrm{V} 180$ adjuvanted with Alhydrogel ${ }^{\mathrm{TM}}$ (aluminum hydroxide gel, Brenntag Biosector, Frederikssund, Denmark) $(N=8)$, or placebo $(N=4)$. Immunogenicity was measured using a plaque reduction neutralization test at days $1,15,28$, and 180 after vaccination. In addition, vaccine safety (solicited and unsolicited adverse events) was assessed using a vaccination report card for 28 days following vaccination, and serious adverse events were captured from the time of informed consent through the final study visit at 6 months after vaccination. The results of the study demonstrate that the $\mathrm{V} 180$ vaccine is generally well tolerated and immunogenic in these dengue-seropositive volunteers.
\end{abstract}

\section{INTRODUCTION}

Dengue is currently the most prevalent vector-borne viral disease and results in a significant amount of human morbidity and mortality. Underreporting and underdiagnosis of the infection remain a key challenge; however, it is estimated that there are 390 million cases per year, including 96 million cases with clinical symptoms ${ }^{1}$ and approximately 10,000 denguerelated deaths. ${ }^{2}$ Dengue infection can present a wide range of clinical symptoms ranging from mild febrile illness to a severe life-threatening syndrome that includes hemorrhagic complications, plasma leakage, and circulatory collapse. Treatment is limited to supportive care.

Dengue virus (DENV) is a member of the family Flaviviridae with four distinct serotypes, DENV1-4. These four serotypes are diverse in sequence, particularly in the viral envelope, the primary target of neutralizing antibodies. Infection with one serotype provides durable homotypic protection ${ }^{3}$ with a period of heterotypic cross-protection against symptomatic infection with other serotypes for a period of time, ranging from a few months up to 2 years. ${ }^{4-6}$ After this short-term heterotypic immunity wanes, infection with a different serotype is a risk factor for severe disease. ${ }^{7}$ The immune correlates of protection for dengue viral immunity are likely complex and not yet precisely defined ${ }^{8}$; however, high levels of serum neutralization titers are believed to be correlated with protection

\footnotetext{
*Address correspondence to Stephen S. Whitehead, Laboratory of Viral Diseases, National Institute of Allergy and Infectious Diseases, National Institutes of Health, Bldg. 33, Rm. 3W10A, 33 North Dr.,
} Bethesda, MD 20892. E-mail: swhitehead@niaid.nih.gov from symptomatic infection. ${ }^{9,10}$ Current DENV vaccine efforts are focused on candidates inducing durable and balanced type-specific neutralizing antibody responses to each serotype.

To date, one dengue vaccine, Dengvaxia ${ }^{\circledR}$ (Sanofi Pasteur, Lyon, France), is licensed in several countries for individuals living in endemic areas. Despite efficacy in dengue-experienced individuals, longer term safety data demonstrated that this vaccine increased the risk of more severe symptoms on DENV infection in seronegative subjects and children younger than 9 years, ${ }^{11-13}$ thereby limiting its use. Moreover, in 2019, the FDA approved the vaccine for use in endemic parts of the United States and only in individuals aged 9-16 years with a previous laboratory-confirmed dengue infection. New DENV vaccines are in development to address these limitations and unmet medical needs.

The live attenuated tetravalent vaccine (LATV) developed at the National Institute of Allergy and Infectious Diseases is concurrently undergoing clinical development in a number of trials. The vaccine comprises four recombinant live attenuated DENV components designed with molecularly defined attenuating mutations. ${ }^{14}$ Two formulations (TV003 and TV005) of the LATV candidate have been clinically tested and have been shown to induce trivalent or tetravalent neutralizing antibody responses in the vast majority participants after a single primary dose. ${ }^{15,16}$ TV003 and TV005 are identical in formulation, with the exception that the DENV2 component is given at a 10fold higher dose in TV005. Currently, it is unknown whether a booster vaccination will be required, and clinical studies are ongoing to address this question. Administration of a second dose of TV003 or TV005 vaccines has not been associated 
with significant increases in neutralizing antibody titers. ${ }^{15-17}$ Therefore, we were interested in evaluating an alternative boosting regimen. Recombinant subunit protein vaccines may offer advantages for this purpose such as the ability to boost immune responses in the presence of preexisting immunity or to more narrowly target the immune response toward an immunologically important antigen.

In the current study, a booster immunization with an investigational tetravalent dengue subunit vaccine, V180, a formulation comprising a recombinant truncated envelope protein (DEN-80E) for each of the four DENV serotypes, was evaluated. ${ }^{18}$ Data from two previous clinical trials demonstrated that Alhydrogel-adjuvanted formulations of V180 were generally well tolerated and induced a modest immune response in Flavivirus-naive individuals ${ }^{19,20}$ (NCT00936429 and NCT01477580). The purpose of this proof-of-principle study (NCT02450838) was to assess the safety and tolerability of unadjuvanted V180 or V180 formulated with Alhydrogel and to determine if the recombinant $\mathrm{V} 180$ vaccines could boost the neutralizing antibody response to DENV1, DENV2, DENV3, and DENV4 when administered to volunteers who had previously received the live attenuated tetravalent dengue vaccine formulations TV003 or TV005.

\section{MATERIALS AND METHODS}

Study design. This was a phase-1, randomized, placebocontrolled, double-blind study that evaluated the safety, tolerability, and immunogenicity of a tetravalent recombinant subunit dengue vaccine (protocol \#V180-002 [CIR-301]) in healthy adults aged 18-50 years who previously received an investigational live attenuated tetravalent dengue vaccine, either TV003 or TV005, and responded (seroconverted as measured by the plaque reduction neutralization test using a $50 \%$ neutralization cutoff) $\left(\mathrm{PRNT}_{50}\right.$ titer $\geq 10$ ) to $\geq 3$ vaccine serotypes. The study was conducted at two sites in the United States, Baltimore, MD, and Burlington, VT, between April and October 2015. Dengue virus is not endemic to these areas. Participants who met the entry criteria were randomized to receive one intramuscular injection of V180 adjuvanted with Alhydrogel, V180 nonadjuvanted, or placebo at 2:2:1. After vaccination, participants were evaluated in the clinic for at least 30 minutes and then returned to the clinic on days 15,28 , and 180 . In addition to study visits, the participants were contacted by telephone at 7 days and 3 months after vaccination to review any adverse events (AEs) and serious adverse events (SAEs) that may have occurred following vaccination. Each volunteer participated in the study for approximately 26 weeks, from the time of vaccination through the final contact (approximately 180 days following vaccination). An external, independent, and blinded data safety monitoring board reviewed the trial design before initiation and reviewed data during the trial to evaluate the safety, study progress, and conduct of the study.

Population. Adult males or females in generally good health between 18 and 50 years of age, inclusive, who had previously received the TV003 or TV005 LATV dengue vaccine formulation successfully completed the NIH study of TV003 or TV005 (NCT01436422 or NCT01072786) ${ }^{16,21}$ with no safety concerns, and had seroconverted for $\geq 3$ serotypes were eligible for enrollment. Before the TV003 or TV005 vaccinations, all participants were Flavivirus naive (i.e., seronegative for all
DENV serotypes, yellow fever virus, West Nile virus, and St. Louis encephalitis virus).

The requirement for prior seroconversion for $\geq 3$ serotypes was designed to evaluate the ability of V180 to boost in the face of a robust response that had already been induced by LATV - a situation in which it is very difficult to boost with an additional dose of LATV. ${ }^{15-17}$ Good general health was determined by physical examination, laboratory screening, and a review of medical history. The number of LATV doses previously received (1 or 2) and the time from the last dose of LATV were not controlled beyond the requirement that a minimum of 6 months must have passed since the subject received the last dose of LATV.

Participants were excluded if they had potential or known exposure to Flavivirus by infection or vaccination in the interim since the receipt of the last dose of the experimental dengue vaccine (TV003 or TV005) and planned travel to a DENVendemic area in the 30 days before or anytime during the current study. Other exclusion criteria included evidence of clinically significant neurologic, cardiac, pulmonary, hepatic, rheumatologic, autoimmune, or renal disease; poorly controlled diabetes mellitus; recent receipt of blood products; febrile illness $\leq 72$ hours before the first dose; and receipt of any licensed vaccine within 14 or 30 days, respectively, before vaccination in this study.

This study was conducted in conformance with the ethical principles originating from the Declaration of Helsinki, Good Clinical Practice requirements, including obtaining written informed consent from each participant before the study entry, and was approved by the human studies committees of each study site.

Vaccines. V180 is a candidate tetravalent vaccine comprising truncated recombinant DENV envelope glycoproteins (E) consisting of the $N$-terminal $80 \%$ of $E$ (DEN-80E) for each DENV serotype. ${ }^{18}$ The vaccines were administered at a final dose of $10 \mu \mathrm{g}$ DEN1-80E, $10 \mu \mathrm{g}$ DEN2-80E, $10 \mu \mathrm{g}$ DEN3-80E, and $20 \mu \mathrm{g}$ DEN4-80E. The higher dose of DEN4-80E was used because of reduced immunogenicity as compared with the other serotypes observed in previous studies. ${ }^{20,22}$ The inclusion of Alhydrogel was evaluated at a final dose of $0.23 \mathrm{mg}$. The V180 and the V180 plus Alhydrogel formulations were compared with a placebo group, which consisted of vaccination with $1 \times$ Dulbecco's phosphate-buffered saline.

All products were prepared, packaged, and labeled according to good manufacturing practice, guidelines from the International Conference on Harmonization of Technical Requirements for Registration of Pharmaceuticals for Human Use, and applicable local laws and regulations. Vaccine supplies were shipped, stored, and distributed in accordance with the trial protocol.

Safety objectives. A primary objective of the study was to assess the overall safety and tolerability of the tested V180 formulations when administered to healthy adults aged 18-50 years. Adverse events were recorded using a standardized vaccination report card for 14 days after vaccination. The secondary safety objectives of this study were to provide a safety summary for each vaccination group. Solicited injection-site AEs (redness, swelling, and pain/tenderness) of any size or intensity from day 1 to day 5 were collected. The participants were additionally surveyed from days 1 to 14 after vaccination for the following: unsolicited injection-site AEs, maximum reported temperature, solicited systemic AEs (fatigue, myalgia, and headache), and unsolicited systemic AEs. Data for any 
serious AEs including deaths from the time of informed consent through approximately day 180 and for medically attended AEs from day 15 through approximately day 180 were also collected.

Safety data analysis. The overall safety and tolerability of the tested V180 formulations were assessed and reported as a summary of serious and nonserious solicited and unsolicited AEs. A formal hypothesis was not tested.

Immunogenicity objectives. The primary immunogenicity objective of the trial was to evaluate if at least one of the V180 formulations induced a booster-neutralizing antibody response, as measured by the $\mathrm{PRNT}_{50}$, to at least three of the four DENV serotypes at day 28 after vaccination. For each V180 formulation, the quantitative criteria for a positive booster immune response was that the geometric mean $\mathrm{PRNT}_{50}$ titer of the group at 28 days after vaccination was $\geq 150$, and that the geometric mean fold rise (GMFR) at 28 days after vaccination was $\geq 3$ ater vaccination when compared with prevaccination for at least three of the four serotypes. A 3-fold rise in neutralization was selected because that value is greater than the variability of the neutralization assay, which is approximately 2-fold, and it was considered that a 3-fold increase could be clinically meaningful. A specific immunogenicity hypothesis was not tested. Exploratory immunogenicity objectives included analysis of the kinetics and durability of the virus neutralizing response using the $\mathrm{PRNT}_{50}$ performed at days 15 and 180 as well as analysis of the neutralization response using the focus reduction neutralization test (FRNT ${ }_{50}$ ) at days 28 and 180 after vaccination.

Immunogenicity assessments. Blood samples for the assessment of immune responses were collected from all participants before vaccination on day 1 and at days 15, 28, and 180 after vaccination. The samples were evaluated for DENV1-, DENV2-, DENV3-, and DENV4-neutralizing antibody by the plaque reduction neutralization test using a $50 \%$ neutralization cutoff $\left(\mathrm{PRNT}_{50}\right)$ with the testing conducted at Johns
Hopkins University or the University of Vermont using the procedure explained in the following text. The $\mathrm{FRNT}_{50}$ conducted at Focus Diagnostics (as explained in the following text) was used as an exploratory immunogenicity measurement to collect the same immunogenicity output as previous Merck \& Co., Inc., (Kenilworth, NJ) dengue vaccine studies.

Plaque reduction neutralization test. Serum antibody levels of DENV1, DENV2, DENV3, and DENV4 were measured by $\mathrm{PRNT}_{50}$ as previously described. ${ }^{23}$ Briefly, the $\mathrm{PRNT}_{50}$ was performed by titrating serum and controls, tested in duplicate, and then adding an equal volume of DENV (DENV1 Western Pacific, DENV2 New Guinea C, DENV3 Sleman/78, or DENV4 Dominica/81) containing a consistent target amount of infectious virus for each given strain. The DENV strains are heterologous to the V180 parental strains for all four DENV serotypes. A collection of human sera which was positive for each of the four dengue serotypes was used for the positive control. After an incubation period that allows for virus neutralization, each serum/virus mix was then transferred to a 24-well plate containing confluent Vero cells (the WHO origin). Following incubation at $37^{\circ} \mathrm{C}$ to allow non-neutralized virus absorbance, overlay medium was applied to immobilize the virus. Plates were incubated for 4 days to allow for growth of virus in infected cells. Plates were then fixed with methanol and infectious cell plaques in the monolayer and were detected by an immunostain using specific anti-dengue mouse monoclonal antibodies, a secondary antibody labeled with peroxidase, and a precipitating substrate. The titer of a sample was determined by counting the infectious plaques (foci). The antibody titer resulting from the PRNT 50 is defined as the highest dilution of antibody that reduces the number of plaques by at least $50 \%$, compared with the plaque titer of virus in the negative control.

Focus reduction neutralization test. The $\mathrm{FRNT}_{50}$ (Focus Diagnostics, Cyrus, CA) is performed by titrating serum and controls, tested in duplicate, and then adding an equal volume

TABLE 1

Subject baseline characteristics

\begin{tabular}{|c|c|c|c|c|c|c|}
\hline & \multicolumn{2}{|c|}{ V180 + Alhydrogel } & \multicolumn{2}{|c|}{ Unadjuvanted V180 } & \multicolumn{2}{|c|}{ Placebo } \\
\hline & $n$ & (\%) & $n$ & (\%) & $n$ & (\%) \\
\hline Subjects in population & 8 & & 8 & & 4 & \\
\hline \multicolumn{7}{|l|}{ Gender } \\
\hline Male & 6 & $(75.0)$ & 5 & $(62.5)$ & 2 & $(50.0)$ \\
\hline Female & 2 & (25.0) & 3 & (37.5) & 2 & (50.0) \\
\hline \multicolumn{7}{|l|}{ Age (years) } \\
\hline$<18$ & 0 & $(0.0)$ & 0 & $(0.0)$ & 0 & $(0.0)$ \\
\hline $18-27$ & 3 & (37.5) & 2 & $(25.0)$ & 1 & $(25.0)$ \\
\hline $28-37$ & 3 & (37.5) & 3 & (37.5) & 2 & (50.0) \\
\hline $38-45$ & 2 & (25.0) & 2 & (25.0) & 0 & $(0.0)$ \\
\hline $46-49$ & 0 & $(0.0)$ & 1 & (12.5) & 1 & $(25.0)$ \\
\hline$\geq 50$ & 0 & $(0.0)$ & 0 & $(0.0)$ & 0 & $(0.0)$ \\
\hline Mean & 30.5 & & 34.1 & & 34.5 & \\
\hline SD & 7.5 & & 8.4 & & 10.1 & \\
\hline Median & 30.5 & & 33.0 & & 31.5 & \\
\hline Range & $22-41$ & & $23-47$ & & $26-49$ & \\
\hline \multicolumn{7}{|l|}{ Race } \\
\hline Black or African American & 4 & $(50.0)$ & 4 & $(50.0)$ & 2 & $(50.0)$ \\
\hline Multiple & 1 & (12.5) & 1 & (12.5) & 0 & $(0.0)$ \\
\hline White & 3 & $(37.5)$ & 3 & $(37.5)$ & 2 & $(50.0)$ \\
\hline \multicolumn{7}{|l|}{ Ethnicity } \\
\hline Hispanic or Latino & 0 & $(0.0)$ & 1 & $(12.5)$ & 0 & $(0.0)$ \\
\hline Not Hispanic or Latino & 8 & (100.0) & 6 & (75.0) & 4 & (100.0) \\
\hline Unknown & 0 & $(0.0)$ & 1 & (12.5) & 0 & $(0.0)$ \\
\hline
\end{tabular}


of DENV (DENV1 WHO WestPac 74, DENV2 WHO S16803, DENV3 WHO CH53489, or DENV4 WHO TVP360) containing a consistent target amount of infectious virus for each given strain. The DENV strains are heterologous to the V180 parental strains for DENV1, DENV2, and DENV4, and homologous for the V180 DENV3 component. After an incubation period at $37^{\circ} \mathrm{C}$ for virus neutralization, each serum/virus mix is then transferred to a 24-well plate containing confluent Vero cells (WHO origin). A collection of human sera which was positive for each of the four dengue serotypes was used for the positive control. Following incubation at $37^{\circ} \mathrm{C}$ to allow non-neutralized virus absorbance, overlay medium is applied to immobilize virus. An incubation period for a specified number of days, specific for each DENV serotype, occurs to allow for growth of virus in infected cells. Plates are fixed with formaldehyde, and infectious foci in the cell layer are detected by immunostaining using a specific anti-dengue rabbit antibody, a secondary antibody labeled with peroxidase, and a precipitating substrate. The titer of a sample is determined by counting the infectious foci. The results are reported as an $\mathrm{FRNT}_{50}$ titer, the highest dilution of antibody that results in a $50 \%$ reduction in the number of infectious foci. The $\mathrm{FRNT}_{50}$ antibody titer is calculated by comparing the number of infectious foci in the presence of the test serum with the number of foci present in
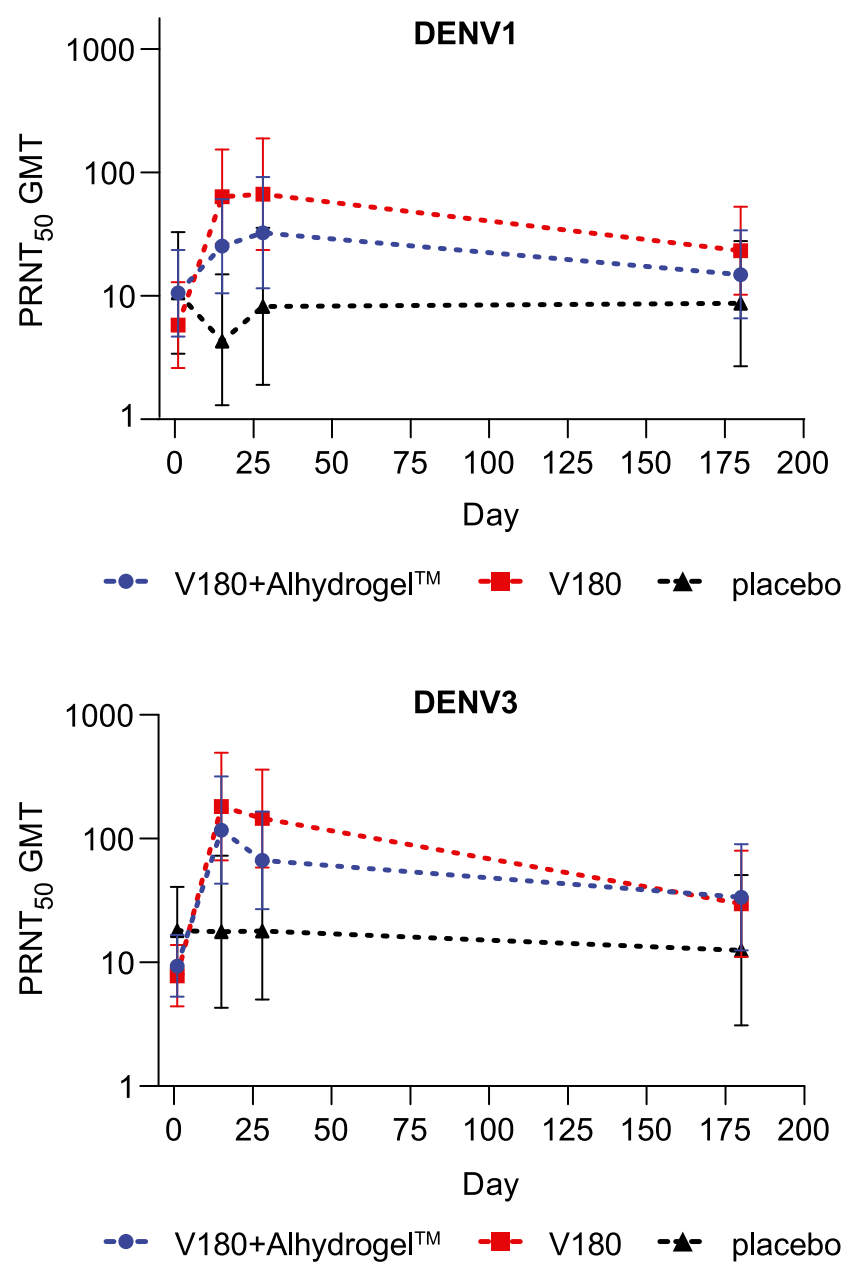

the negative control. The signals from the serial dilutions are fit using a 4-parameter nonlinear logistic curve, which is then used to interpolate the dilution resulting in $50 \%$ reduction.

\section{RESULTS}

Participant accounting and demographics. A total of 31 volunteers were screened for the potential to participate in this study, and 20 were randomized to receive either $\mathrm{V} 180$ adjuvanted with Alhydrogel (V180 + Alhydrogel; $n=8)$, V180 nonadjuvanted $(n=8)$, or placebo $(n=4)$ at 2:2:1. Eleven subjects were screened but not randomized because of the exclusion criteria. All randomized participants received one intramuscular injection of study vaccine or placebo and completed the follow-up visits for safety and immunogenicity through day 180. Gender, age, race, and ethnicity were generally consistent across the treatment groups (Table 1). The time since LATV ranged from 1.9 to 3.8 years.

Immunogenicity. Serum neutralization titers against all four DENV serotypes were measured at days 1, 15, 28, and 180 after vaccination using a PRNT with a $50 \%$ neutralization cutoff. After vaccination, the geometric mean titers (GMTs) of neutralizing antibody for the groups given V180 + Alhydrogel or unadjuvanted V180 increased compared with the day 1
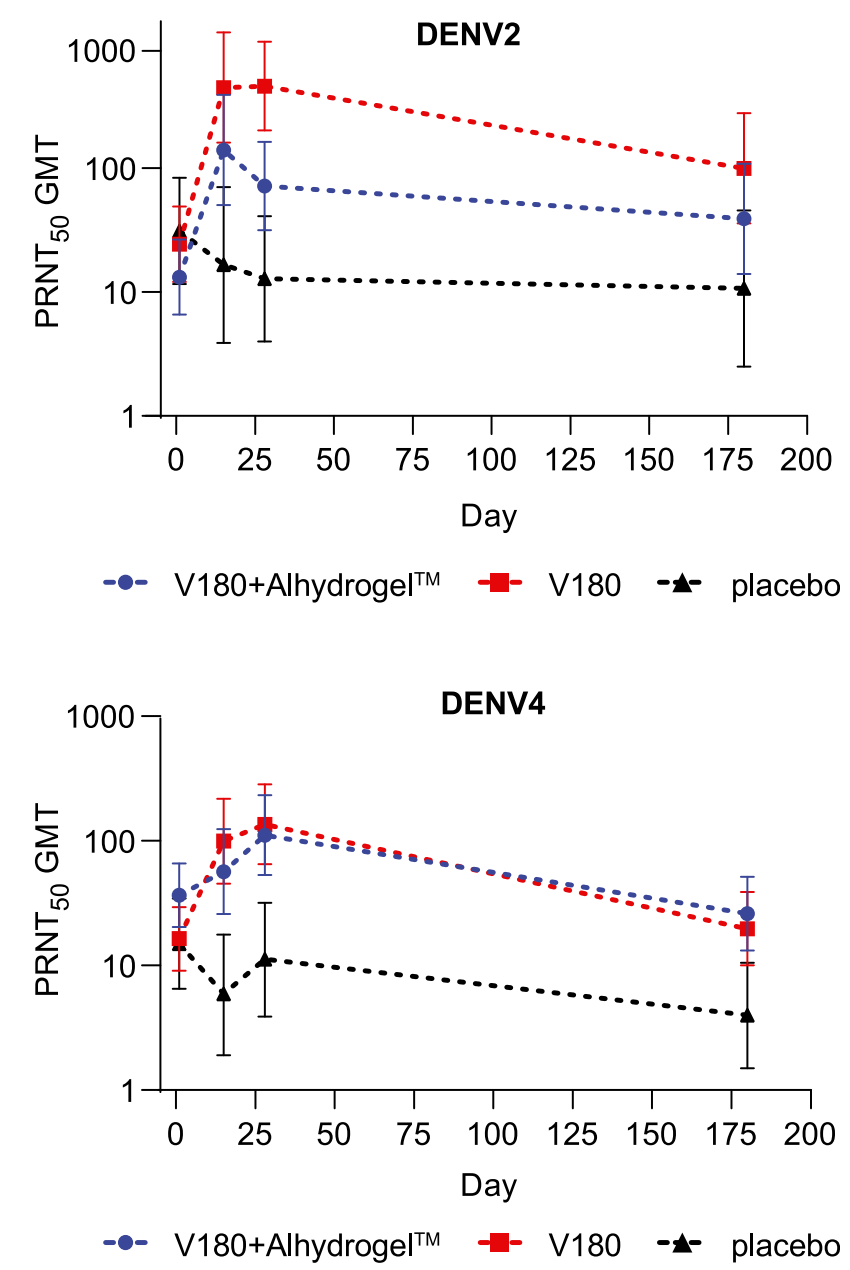

FIGURE 1. Longitudinal immune responses as measured by PRNT 50 . The geometric mean titer (GMT) of neutralizing antibody of each group is shown at days 1, 15, 28, and 180 for DENV1 (A), DENV2 (B), DENV3 (C), DENV4 (D) as measured with the plaque reduction neutralization test $\left(\mathrm{PRNT}_{50}\right)$. Error bars represent the $95 \% \mathrm{Cl}$ for each group. This figure appears in color at www.ajtmh.org. 
GMT for all four serotypes (Figure 1), demonstrating that immunization with V180 increased immune responses induced by LATV. In terms of magnitude, we predesignated a GMT $\geq 150$ at day 28 in at least three of four serotypes as a criterion of a positive immune response. The GMTs in the V180 + Alhydrogel group were all less than this threshold. (Figure 1, Table 2). However, in the unadjuvanted V180 group, DENV2 responses were $\geq 150$ at day 28 , and responses to DENV3 and DENV4 were approaching that level, yet under the designated cutoff (Figure 1, Table 2). A trend of higher peak neutralization titers was observed in the V180 group than in the V180 + Alhydrogel group (Figure 1, Table 2).

We also analyzed the day $28 \mathrm{PRNT}_{50}$ for the GMFR over day 1 (Table 2). Viewing the data this way demonstrated that V180 increased the serum neutralization titers in the groups vaccinated with either V180 formulation. Specifically, V180 adjuvanted with Alhydrogel induced a GMFR (day 28/day 1 ) ranging from 3.0- to 7.2-fold across serotypes, and unadjuvanted V180 induced a GMFR ranging from 8.3- to 18.8-fold (Table 2). By contrast, the placebo group fold change (day 28 /day 1 ) remained near to 1 (range 0.4-1.0) across DENV serotypes (Table 2) because DENV does not circulate naturally in the areas of the study.

Serum neutralization titers were also analyzed at day 180 (6 months) after vaccination to evaluate the durability of response. At day 180, neutralization titers for all four serotypes had declined in the V180 vaccinated groups as compared with the responses measured at day 28 (Figure 1). Yet, compared with baseline measurements, titers were still elevated in some serotypes at this later timepoint. The GMFR of the V180+ Alhydrogel group were 1.4, 3.0, 3.6, and 0.7 (day 180/day 1) for DENV1, DENV2, DENV3, and DENV4, respectively. (Supplemental Table 1). In addition, the unadjuvanted V180 vaccinated group demonstrated a 4.0, 4.1, 3.8, and 1.2 geometric mean fold rise (day 180/day 1) for DENV1, DENV2, DENV3, and DENV4, respectively (Supplemental Table 1). In comparison, the placebo GMFR at day 180 was $0.8,0.3,0.7$, and 0.3 for DENV1, DENV2, DENV3, and DENV4, respectively (Supplemental Table 1).

In addition, we analyzed the $\mathrm{PRNT}_{50}$ data for seropositivity frequencies, where seropositivity was defined as a PRNT 50 antibody titer $\geq 10$. The frequency of seropositivity increased following vaccination with both V180 formulations (Figure 2). Furthermore, these seroconversions were durable; in many cases, the frequencies remained elevated at day $180 \mathrm{com}-$ pared with baseline (Figure 2).

The immunogenicity data overall showed that administration of V180 alone or with Alhydrogel increased DENV-specific serum neutralization titers and seropositivity frequency in participants previously vaccinated with LATV; however, these levels failed to meet the protocol-specified criteria for a positive booster response and declined over time.

Safety. Both V180 formulations were generally well tolerated, and participants assessed most AEs to be of mild or moderate intensity (data not shown). Serious adverse events, deaths, or withdrawals due to AEs were not observed at any time during the study.

In total, for all three vaccine groups, 12 participants reported one or more injection-site and/or systemic AEs from days 1 to 14 after vaccination (Table 3). The incidence of injection-site AEs was higher in the V180 + Alhydrogel group than in the V180 group and the placebo group (Table 4). The most commonly reported injection-site $A E$ was injection-site pain (Table 4).

Systemic AEs in the V180 vaccinated groups were comparable to the placebo group, with fatigue and headache as the most commonly reported events (Supplemental Table 2).

\section{DISCUSSION}

This trial assessed the safety, tolerability, and immunogenicity of a recombinant DENV-envelope vaccine (V180) formulated with or without Alhydrogel in participants who were previously vaccinated with the live attenuated tetravalent dengue vaccines, TV003 or TV005. Before the booster with V180, each of the vaccinees were seropositive to three or more DENV serotypes from previous LATV immunization. This is the first evaluation of V180 in individuals with preexisting DENVneutralizing antibody titers.

Both of the V180 formulations increased the geometric mean neutralization titers (GMTs) (Figure 1) and the frequency of $\mathrm{PRNT}_{50}$ positivity (Figure 2) in these vaccinees. Notably, for

TABLE 2

Geometric mean titer and geometric mean fold ratio of immune responses as measured by $\mathrm{PRNT}_{50}$

\begin{tabular}{|c|c|c|c|c|c|c|}
\hline & \multicolumn{2}{|c|}{ V180 + Alhydrogel $(N=8)$} & \multicolumn{2}{|c|}{ Unadjuvanted V180 $(N=8)$} & \multicolumn{2}{|c|}{ Placebo $(N=4)$} \\
\hline & Observed response & $95 \% \mathrm{Cl}^{*}$ & Observed response & $95 \% \mathrm{Cl}^{\star}$ & Observed response & $95 \% \mathrm{Cl}^{*}$ \\
\hline \multicolumn{7}{|l|}{ DENV1 } \\
\hline Pre-V180 booster GMT (day 1) & 10.6 & $(4.7,23.6)$ & 5.8 & $(2.6,12.9)$ & 10.6 & $(3.4,32.9)$ \\
\hline Post-V180 booster GMT (day 28) & 32.5 & $(11.5,91.8)$ & 66.7 & $(23.6,188.5)$ & 8.2 & $(1.9,35.7)$ \\
\hline GMFR (day 28) & 3.1 & $(1.4,6.8)$ & 11.6 & $(5.2,25.4)$ & 0.8 & $(0.3,2.4)$ \\
\hline \multicolumn{7}{|l|}{ DENV2 } \\
\hline Pre-V180 booster GMT (day 1 ) & 13.2 & $(6.6,26.6)$ & 24.4 & $(12.1,49.0)$ & 31.2 & $(11.6,83.9)$ \\
\hline Post-V180 booster GMT (day 28) & 71.8 & $(31.5,163.4)$ & 459.6 & $(201.9,1,046.2)$ & 12.8 & $(4.0,41.1)$ \\
\hline GMFR (day 28 ) & 5.4 & $(2.8,10.4)$ & 18.8 & $(9.8,36.1)$ & 0.4 & $(0.2,1.0)$ \\
\hline \multicolumn{7}{|l|}{ DENV3 } \\
\hline Pre-V180 booster GMT (day 1) & 9.3 & $(5.3,16.6)$ & 7.8 & $(4.4,13.8)$ & 18.0 & $(8.0,40.6)$ \\
\hline Post-V180 booster GMT (day 28) & 66.8 & $(26.9,165.5)$ & 145.1 & $(58.5,359.7)$ & 17.9 & $(5.0,64.8)$ \\
\hline GMFR (day 28) & 7.2 & $(3.9,13.2)$ & 18.7 & $(10.1,34.3)$ & 1.0 & $(0.4,2.4)$ \\
\hline \multicolumn{7}{|l|}{ DENV4 } \\
\hline Pre-V180 booster GMT (day 1) & 36.6 & $(20.4,65.8)$ & 16.4 & $(9.1,29.4)$ & 15.0 & $(6.5,34.4)$ \\
\hline Post-V180 booster GMT (day 28) & 111.0 & $(53.0,232.2)$ & 135.5 & $(64.8,283.6)$ & 11.2 & $(3.9,31.8)$ \\
\hline GMFR (day 28) & 3.0 & $(1.8,5.0)$ & 8.3 & $(5.0,13.7)$ & 0.7 & $(0.4,1.5)$ \\
\hline
\end{tabular}

fubjects randomized to the respective vaccination ifgroup who received a booster vaccination; $\mathrm{PRNT}_{50}=$ plaque reduction neutralization test with $50 \%$ neutralization cutoff. GMFR measures from pre-V180 booster to day 28 .

$\star 95 \% \mathrm{Cl}$ is based on the Clopper-Pearson exact method. 
DENV1

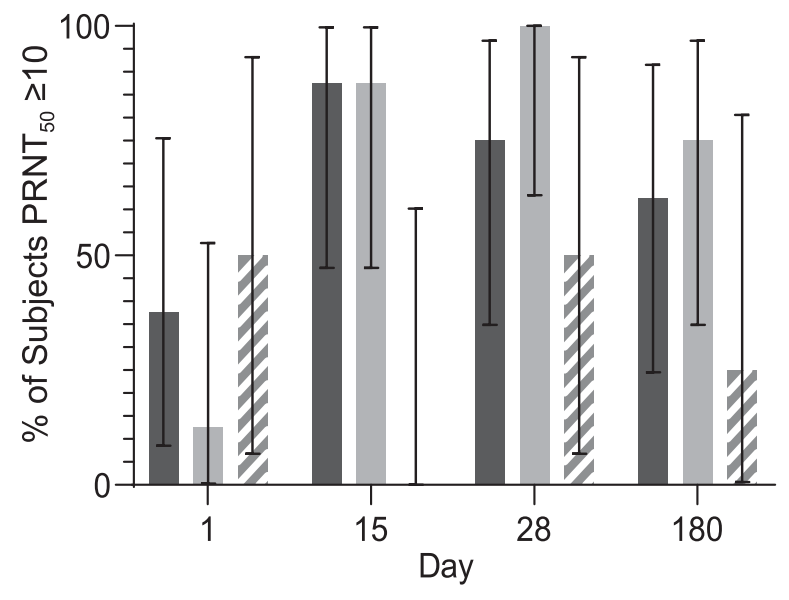

V180+Alhydroge $\mathrm{I}^{\mathrm{TM}} \mathrm{V} 180 \quad \mathrm{~V} / \mathrm{placebo}$

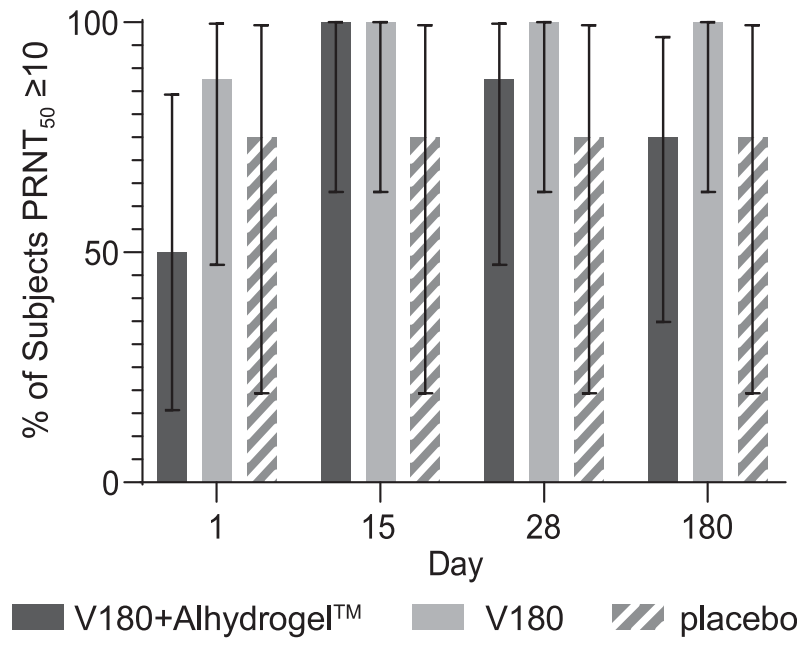

DENV4

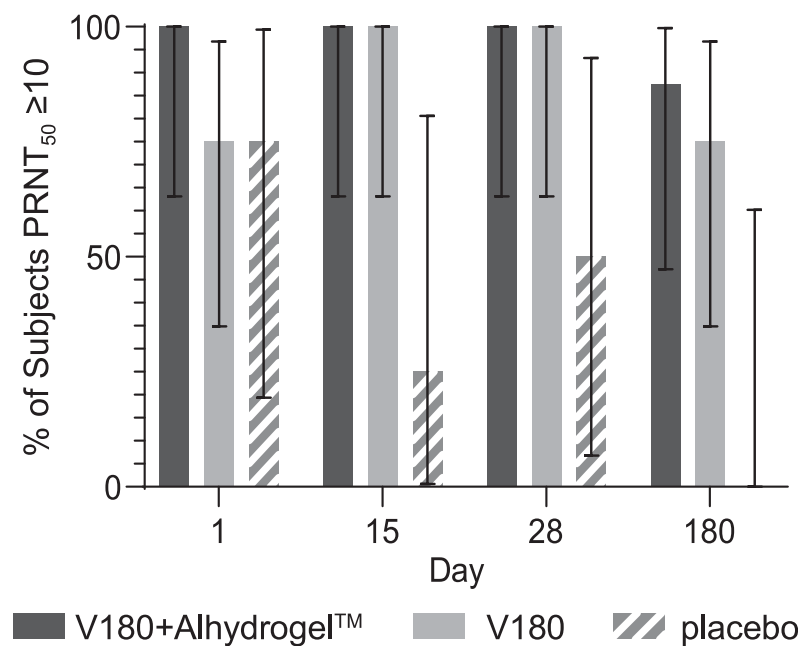

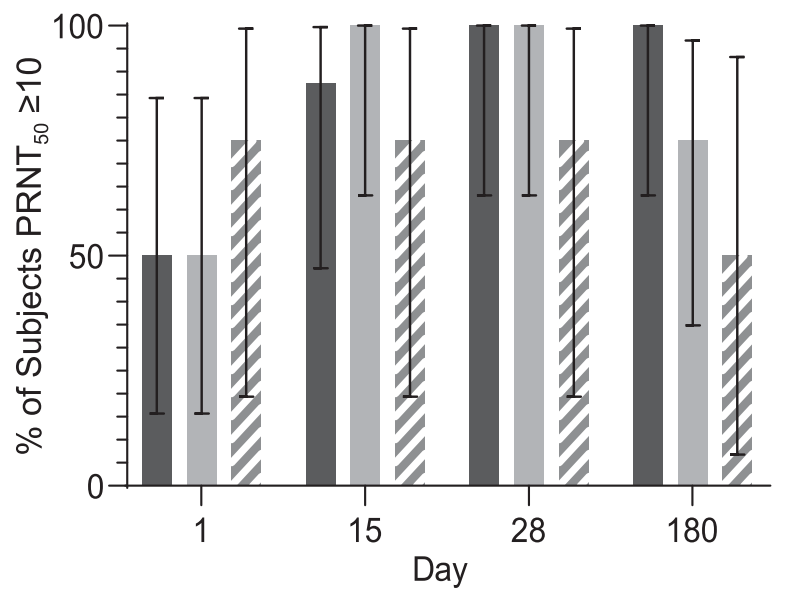

V180+Alhydrogel ${ }^{\mathrm{TM}} \quad$ V180 VI/ placebo

FIGURE 2. Longitudinal plaque reduction neutralization test (PRNT 50 ) seropositivity frequency. The frequency of seropositivity for each group at days $1,15,28$, and 180 is shown for DENV1 (A), DENV2 (B), DENV3 (C), and DENV 4 (D). Seropositivity was defined as a PRNT $50 \geq 10$. Error bars represent the $95 \% \mathrm{Cl}$ for each group.

all four DENV serotypes, the geometric mean fold rises in the unadjuvanted V180 and the V180+ Alhydrogel were greater than 3-fold higher at day 28 than day 1 , ranging from 3.0- to 7.2-fold in the adjuvanted neutralizing group and 8.3- to 18.8-fold in the unadjuvanted group (Table 2). The magnitudes of these titers at day 28 did not reach a group GMT $\geq 150$, with the exception of the response to DENV2 in the V180 group; therefore, the protocol-specified immunogenicity criteria for the trial were not met. Interestingly, the GMTs in the unadjuvanted V180 group for DENV3 and DENV4 were quite close to the cutoff, at 145.1 and 135.6, respectively (Table 2 ). It is currently unknown whether this level of increase in antibody titer would offer any clinical benefit because precise levels of neutralizing antibodies needed for protection from dengue are not yet defined for these vaccines.

A trend of higher immunogenicity in the unadjuvanted V180 group than in the V180 + Alhydrogel group was an unexpected finding. Aluminum-based adjuvants remain the most commonly used adjuvant for human vaccines and have been shown to enhance humoral immunity across numerous antigens, including several licensed protein subunit-based vaccines. ${ }^{24}$ A previous study of V180 in Flavivirus-naive subjects by Manoff et al. ${ }^{20}$ reported that after the third dose, the group administered V180 + Alhydrogel showed slightly increased GMTs for DENV1, DENV2, and DENV3 serotypes compared with the unadjuvanted V180 group, although the titers were very low in both groups. In the current study, although the point estimate for the GMT at day 28 in the unadjuvanted V180 group is numerically higher than those observed in the V180 + Alhydrogel group for all serotypes, the $95 \% \mathrm{Cls}$ of the GMTs at day 28 in the unadjuvanted V180 and the V180 + Alhydrogel overlap for DENV1, DENV3, and DENV4 (Table 2). Here, we are limited by the small number of study participants, and it is unknown whether this trend would be reproduced in a larger study.

The levels of immunity reported here should also be evaluated in the context of previous studies in which participants 
TABLE 3

Adverse even summary days 1-14 after vaccination

\begin{tabular}{|c|c|c|c|c|c|c|}
\hline & \multicolumn{2}{|c|}{ V180 + Alhydrogel } & \multicolumn{2}{|c|}{ Unadjuvanted V180 } & \multicolumn{2}{|c|}{ Placebo } \\
\hline & $n$ & (\%) & $n$ & (\%) & $n$ & (\%) \\
\hline Subjects in population with follow-up & 8 & & 8 & & 4 & \\
\hline With one or more adverse events & 6 & (75.0) & 4 & $(50.0)$ & 2 & $(50.0)$ \\
\hline Injection site & 4 & (50.0) & 2 & (25.0) & 1 & $(25.0)$ \\
\hline Non-injection site & 5 & (62.5) & 3 & (37.5) & 2 & $(50.0)$ \\
\hline With no adverse event & 2 & (25.0) & 4 & (50.0) & 2 & $(50.0)$ \\
\hline With vaccine-related ${ }^{\star}$ adverse events & 6 & (75.0) & 3 & $(37.5)$ & 2 & $(50.0)$ \\
\hline Injection site & 4 & (50.0) & 2 & $(25.0)$ & 1 & $(25.0)$ \\
\hline Non-injection site & 4 & $(50.0)$ & 2 & (25.0) & 2 & $(50.0)$ \\
\hline With serious adverse events & 0 & $(0.0)$ & 0 & $(0.0)$ & 0 & $(0.0)$ \\
\hline With serious vaccine-related adverse events & 0 & $(0.0)$ & 0 & $(0.0)$ & 0 & $(0.0)$ \\
\hline Who died & 0 & $(0.0)$ & 0 & $(0.0)$ & 0 & $(0.0)$ \\
\hline Discontinued $\dagger$ because of an adverse event & 0 & $(0.0)$ & 0 & $(0.0)$ & 0 & $(0.0)$ \\
\hline Discontinued because of a vaccine-related adverse event & 0 & $(0.0)$ & 0 & $(0.0)$ & 0 & $(0.0)$ \\
\hline Discontinued because of a serious adverse event & 0 & $(0.0)$ & 0 & $(0.0)$ & 0 & $(0.0)$ \\
\hline Discontinued because of a serious vaccine-related adverse event & 0 & $(0.0)$ & 0 & $(0.0)$ & 0 & $(0.0)$ \\
\hline
\end{tabular}

†Study medication withdrawn.

were boosted with dengue LATV. The immunogenicity of dengue LATV prime followed by a LATV boost at either $6^{16}$ or $12^{17}$ months was reported using the same PRNT 50 used in the current study. Kirkpatrick et al. ${ }^{16}$ reported on Flavivirus-naive participants vaccinated with TV003 and boosted 6 months later with TV005. Durbin et al. ${ }^{17}$ described a 12-month homologous prime-boost regimen using TV003/TV003. In both of these clinical studies, the geometric means of peak neutralization titers increased less than two-fold for each of the four serotypes. These low levels of immunogenicity measured after the 6- or 12-month boosts have been attributed to the inability of the live virus vaccine to replicate in the presence of high levels of existing DENV-neutralizing antibodies. Although existing antibodies could also interfere with the subunit protein immunogenicity to some extent, these effects are minimized as there are no infection or replication steps, offering a potential advantage for the subunit approach.

Here, we are reporting $\geq 3$-fold peak rise in titers for all serotypes in the V180 immunized groups; however, it is difficult to directly compare the immunogenicity across these studies, in part because of the differences in time/boost intervals in the protocols.

In a study previously reported by Manoff et al., ${ }^{20}$ a threedose regimen (days 1, 30, and 60) of V180 with and without Alhydrogel was tested in Flavivirus-naive participants. Two groups in that study (unadjuvanted V180 and V180+ Alhydrogel-medium dose group) were vaccinated with same dose levels as used here, and serum neutralization titers were then measured using a $\mathrm{FRNT}_{50}$. The unadjuvanted V180 medium dose did not induce any detectable neutralization titers in that study. At 28 days after dose 3, the $\mathrm{V} 180$ + Alhydrogel vaccinated group showed a FRNT 50 GMT of 11, 17, 20, and < 10 for DENV1, DENV2, DENV3, and DENV4, respectively. Seropositivity frequencies after the three doses of V180 + Alhydrogel were also quite low and were reported as $37.5 \%, 50 \%, 62.5 \%$, and $25 \%$ for each serotype. ${ }^{20}$ The same FRNT $_{50}$ was also used as an exploratory assay in the current study, and, here, the GMTs from the $\mathrm{FRNT}_{50}$ of the V180 + Alhydrogel group were numerically higher than those reported by Manoff et al. at day $28(119,171,150$, and 116 for DENV1-4, respectively) with seropositivity frequencies of $87 \%, 87 \%, 100 \%$, and $100 \%$ for each (Supplemental Tables 3 and 4).

The immunogenicity evaluations conducted in this trial were limited by a few factors. Primarily, the total number of vaccinees per group was limited because participants were recruited from the previous TV003 and TV005 trials. Second, a precise immune correlate of protection has not yet been defined for dengue, although levels of serum neutralization titers are correlated with protection from symptomatic infection. ${ }^{8}$ Finally, the optimal timing of a priming LATV followed by a boost with recombinant subunit envelope vaccine is currently unknown. Because we recruited participants from the previous LATV trials, the time interval between prime/boost was longer than most multiple-dose vaccine regimens.

TABLE 4

Injection site adverse events, days 1-14 after vaccination

\begin{tabular}{|c|c|c|c|c|c|c|}
\hline & \multicolumn{2}{|c|}{ V180 + Alhydrogel } & \multicolumn{2}{|c|}{ Unadjuvanted V180 } & \multicolumn{2}{|c|}{ Placebo } \\
\hline & $n$ & (\%) & $n$ & (\%) & $n$ & $(\%)$ \\
\hline Subjects in population with follow-up & 8 & & 8 & & 4 & \\
\hline With one or more injection site adverse events & 4 & $(50.0)$ & 2 & $(25.0)$ & 1 & $(25.0)$ \\
\hline With no injection site adverse events & 4 & $(50.0)$ & 6 & $(75.0)$ & 3 & $(75.0)$ \\
\hline General disorders and administration site conditions & 4 & $(50.0)$ & 2 & $(25.0)$ & 1 & $(25.0)$ \\
\hline Injection site erythema & 1 & $(12.5)$ & 1 & $(12.5)$ & 0 & $(0.0)$ \\
\hline Injection site pain & 4 & $(50.0)$ & 0 & $(0.0)$ & 1 & $(25.0)$ \\
\hline Injection site swelling & 2 & $(25.0)$ & 0 & $(0.0)$ & 0 & $(0.0)$ \\
\hline Injection site vesicles & 0 & $(0.0)$ & 1 & (12.5) & 0 & $(0.0)$ \\
\hline
\end{tabular}


Both V180 formulations were generally well tolerated. This finding is in line with previous evaluations of V180 administered to Flavivirus-naive participants and is expected also because of the well-established safety profile of aluminumbased adjuvants. We were limited in sample size for the safety evaluations, and larger sample sizes will be needed to fully characterize the safety profile.

Overall, V180 formulations were found to be well tolerated and were able to increase serum neutralization titers and the frequency of DENV seropositivity in participants previously vaccinated with dengue LATV. The immunogenicity results did not meet the prespecified criteria for a positive booster immune response; however, it is unknown at this time whether the levels of boosting that were observed would offer any clinical benefit. Furthermore, although current studies suggest that LATV is a single-dose vaccine, future research will determine if a booster dose is necessary for enduring protection. ${ }^{17} \mathrm{~A}$ heterologous prime/boost regimen such as presented here would bring additional commercial challenges such as the manufacturing of two products and a more complex dosing regimen; however, it is something that could be further studied if warranted.

Received January 16, 2020. Accepted for publication April 7, 2020.

Published online May 11, 2020.

Note: Supplemental tables appear at www.ajtmh.org.

Acknowledgments: We thank Kara S. Cox, for medical writing support and Karyn Davis, for editorial assistance (both of Merck \& Co., Inc., Kenilworth, NJ). We would like to thank the Office of Clinical Research Policy and Regulatory Operations (OCRPRO) from the National Institute of Allergy and Infectious Diseases for clinical research assistance. We would also like to thank Kevin Russell for critical reading of the manuscript (from Merck \& Co., Inc., Kenilworth, NJ).

Financial support: This study was funded by Merck Sharp \& Dohme Corp., a subsidiary of Merck \& Co., Inc., Kenilworth, NJ, and in part by the Intramural Research Program of the NIAID, NIH.

Disclosures: M. S., A. R, J. M., D. H., J. R. S., A. W.-T. L., and L. W. are employees of Merck Sharp \& Dohme Corp., a subsidiary of Merck \& Co., Inc., Kenilworth, NJ, and may hold stock in Merck \& Co., Inc., Kenilworth, NJ. B. A.-C. is an employee of Merck Sharp \& Dohme Corp., a subsidiary of Merck \& Co., Inc., Kenilworth, NJ, and may hold stock in Merck \& Co., Inc., Kenilworth, NJ. and has three issued patents related to the V180 vaccine. A. P.D. has participated in scientific advisory committee meetings for Merck \& Co., Inc., Kenilworth, NJ. B. D. K. reports grants from the University of Vermont. P. G. reports employment by Johns Hopkins University, MD. while working on the study. B. P.S. reports grants from the NIAID/NIH and obtaining the vaccine from Merck \& Co., Inc., Kenilworth, NJ to perform the study. M. C. reports employment at MMS Holding, Inc., Jackson, NJ on assignment to Merck \& Co., Inc., Kenilworth, NJ.

Authors' addresses: Anna P. Durbin, Palmtama Grier, Beulah P. Sabundayo, and Helen He, Department of International Health, Center for Immunization Research, Johns Hopkins Bloomberg School of Public Health, Baltimore, MD, E-mails: adurbin1@jhu.edu, pgrier2@ jhu.edu, bsabund1@jhu.edu and hhe6@jhu.edu. Kristen K. Pierce and Beth D. Kirkpatrick, Department of Microbiology and Molecular Genetics, Vaccine Testing Center, Larner College of Medicine, University of Vermont, Burlington, VT, and Department of Medicine, Vaccine Testing Center, Larner College of Medicine, University of Vermont, Burlington, VT, E-mails: kristen.pierce@uvmhealth.org and beth.kirkpatrick@med.uvm.edu. Michele Sausser, Amy Falk Russell, Jason Martin, Donna Hyatt, Melissa Cook, Jeffrey R. Sachs, Andrew Wen-Tseng Lee, Liman Wang, and Beth-Ann Coller, Merck \& Co., Inc., Kenilworth, NJ, E-mails: michele_coia@merck.com, amy_russell@ merck.com, jason_c_martin@merck.com, donna_hyatt@merck.com, melissa.hoffman.cook1@merck.com, jeff_sachs@merck.com, andrew_ wen-tseng_lee@merck.com, liman_wang@merck.com, and beth- ann.coller@merck.com. Stephen S. Whitehead, Laboratory of Viral Diseases, National Institute of Allergy and Infectious Diseases, National Institutes of Health, Bethesda, MD, E-mail: swhitehead@ niaid.nih.gov.

\section{REFERENCES}

1. Bhatt $S$ et al., 2013. The global distribution and burden of dengue. Nature 496: 504-507.

2. Stanaway JD et al., 2016. The global burden of dengue: an analysis from the global burden of disease study 2013. Lancet Infect Dis 16: 712-723.

3. Gibbons RV, Kalanarooj S, Jarman RG, Nisalak A, Vaughn DW, Endy TP, Mammen MP Jr., Srikiatkhachorn A, 2007. Analysis of repeat hospital admissions for dengue to estimate the frequency of third or fourth dengue infections resulting in admissions and dengue hemorrhagic fever, and serotype sequences. Am J Trop Med Hyg 77: 910-913.

4. Reich NG, Shrestha S, King AA, Rohani P, Lessler J, Kalayanarooj S, Yoon IK, Gibbons RV, Burke DS, Cummings DA, 2013. Interactions between serotypes of dengue highlight epidemiological impact of cross-immunity. J R Soc Interf 10: 20130414.

5. Montoya M, Gresh L, Mercado JC, Williams KL, Vargas MJ, Gutierrez G, Kuan G, Gordon A, Balmaseda A, Harris E, 2013. Symptomatic versus inapparent outcome in repeat dengue virus infections is influenced by the time interval between infections and study year. PLoS Negl Trop Dis 7: e2357.

6. Anderson KB et al., 2014. A shorter time interval between first and second dengue infections is associated with protection from clinical illness in a school-based cohort in Thailand. J Infect Dis 209: 360-368.

7. Sangkawibha N, Rojanasuphot $S$, Ahandrik $S$, Viriyapongse $S$, Jatanasen S, Salitul V, Phanthumachinda B, Halstead SB, 1984. Risk factors in dengue shock syndrome: a prospective epidemiologic study in Rayong, Thailand. I. The 1980 outbreak. Am J Epidemiol 120: 653-669.

8. Katzelnick LC, Harris E, 2017. Immune correlates of protection for dengue: state of the art and research agenda. Vaccine 35 : 4659-4669.

9. Katzelnick LC, Montoya M, Gresh L, Balmaseda A, Harris E, 2016. Neutralizing antibody titers against dengue virus correlate with protection from symptomatic infection in a longitudinal cohort. Proc Natl Acad Sci U S A 113: 728-733.

10. Buddhari $D$ et al., 2014. Dengue virus neutralizing antibody levels associated with protection from infection in Thai cluster studies. PLoS Negl Trop Dis 8: e3230.

11. Halstead SB, 2018. Safety issues from a phase 3 clinical trial of a live-attenuated chimeric yellow fever tetravalent dengue vaccine. Hum Vaccin Immunother 14: 2158-2162.

12. Hadinegoro SR et al., 2015. Efficacy and long-term safety of a dengue vaccine in regions of endemic disease. $N$ Engl $J$ Med 373: 1195-1206.

13. Gailhardou S, Skipetrova A, Dayan GH, Jezorwski J, Saville M, Van der Vliet D, Wartel TA, 2016. Safety overview of a recombinant live-attenuated tetravalent dengue vaccine: pooled analysis of data from 18 clinical trials. PLoS Negl Trop Dis 10: e0004821.

14. Blaney JE Jr., Durbin AP, Murphy BR, Whitehead SS, 2006. Development of a live attenuated dengue virus vaccine using reverse genetics. Viral Immunol 19: 10-32.

15. Whitehead SS et al., 2017. In a randomized trial, the live attenuated tetravalent dengue vaccine TV003 is well-tolerated and highly immunogenic in subjects with flavivirus exposure prior to vaccination. PLoS Negl Trop Dis 11: e0005584.

16. Kirkpatrick BD et al., 2015. Robust and balanced immune responses to all 4 dengue virus serotypes following administration of a single dose of a live attenuated tetravalent dengue vaccine to healthy, flavivirus-naive adults. $J$ Infect Dis 212: 702-710.

17. Durbin AP et al., 2016. A 12-month-interval dosing study in adults indicates that a single dose of the national institute of allergy and infectious diseases tetravalent dengue vaccine induces a robust neutralizing antibody response. $J$ Infect Dis 214: 832-835.

18. Coller BA, Clements DE, Bett AJ, Sagar SL, Ter Meulen JH, 2011. The development of recombinant subunit envelope-based 
vaccines to protect against dengue virus induced disease. Vaccine 29: 7267-7275.

19. Manoff SB et al., 2015. Preclinical and clinical development of a dengue recombinant subunit vaccine. Vaccine 33: 7126-7134.

20. Manoff SB et al., 2019. Immunogenicity and safety of an investigational tetravalent recombinant subunit vaccine for dengue: results of a phase I randomized clinical trial in flavivirus-naive adults. Hum Vaccin Immunother 15: 2195-2204.

21. Durbin AP et al., 2013. A single dose of any of four different live attenuated tetravalent dengue vaccines is safe and immunogenic in flavivirus-naive adults: a randomized, double-blind clinical trial. J Infect Dis 207: 957-965.
22. Govindarajan D, Meschino S, Guan L, Clements DE, ter Meulen JH, Casimiro DR, Coller BA, Bett AJ, 2015. Preclinical development of a dengue tetravalent recombinant subunit vaccine: immunogenicity and protective efficacy in nonhuman primates. Vaccine 33: 4105-4116.

23. Durbin AP et al., 2001. Attenuation and immunogenicity in humans of a live dengue virus type- 4 vaccine candidate with a 30 nucleotide deletion in its 3'-untranslated region. Am J Trop Med Hyg 65: 405-413.

24. HogenEsch H, O'Hagan DT, Fox CB, 2018. Optimizing the utilization of aluminum adjuvants in vaccines: you might just get what you want. NPJ Vaccines 3: 51. 